\title{
Locating Faults in MANET-Hosted Software Systems
}

\author{
Petr Novotny, Bong Jun Ko, and Alexander L. Wolf, Fellow, IEEE
}

\begin{abstract}
We present a method to locate faults in service-based software systems hosted on mobile ad hoc networks (MANETs). In such systems, computations are structured as interdependent services distributed across the network, collaborating to satisfy client requests. Faults, which may occur at either or both the service and network layers, propagate by cascading through some subset of the services, from their root causes back to the clients that initiate requests. Fault localization in this environment is especially challenging because the systems are typically subject to a wider variety and higher incidence of faults than those deployed in fixed networks, the resources available to collect and store analysis data are severely limited, and many of the sources of faults are by their nature transient. Our method makes use of service-dependence and fault data that are harvested in the network through decentralized, run-time observations of service interactions and fault symptoms. We have designed timing- and Bayesian-based reasoning techniques to analyze the data in the context of a specific fault propagation model. The analysis provides a ranked list of candidate fault locations. Through extensive simulations, we evaluate the performance of our method in terms of its accuracy in correctly ranking root causes under a wide range of operational conditions.
\end{abstract}

\section{INTRODUCTION}

Mobile ad hoc networks (MANETs) are used in difficult or remote situations, such as emergency response or forestfire fighting, where a reliable, fixed-network communication infrastructure may be absent. However, the applications deployed upon MANETs are increasingly expected to exhibit sophisticated features, mimicking the availability of rich applications in "normal" network environments.

The current trend in software engineering is to architect systems as collections of collaborating software (micro)services, distributed across a network and interacting to satisfy requests, where a request typically originates at a user-facing client [11], [16], [19], [20]. Popular forms of service-based systems include the Service-Oriented Architecture (SOA) and Web Services (WS) frameworks. Figure 1 illustrates a service-based system, with client applications and services deployed across the nodes of a mobile network. The computation required to fulfil a client request results in a cascade of further requests across some subset of the services; services make requests on other services in order to fulfil the requests made upon them. The set of messages exchanged during the processing of a single client request is referred to as a conversation.

We are concerned with a crucial operational aspect of service-based systems deployed on MANETs: locating (also called identifying) a likely root-cause fault within a failed conversation. This is a particularly challenging management task because the systems are subject to a wider variety and higher incidence of faults than those deployed in fixed networks, the resources available to collect and store anal-

P. Novotny and A.L. Wolf are with the Department of Computing, Imperial College London.

B.J. Ko is with the IBM T.J. Watson Research Center.

Manuscript received August 20, 2014.

A preliminary version of this paper appears in the Proceedings of the 31st International Symposium on Reliable Distributed Systems, October 2012, pp. 243-248.

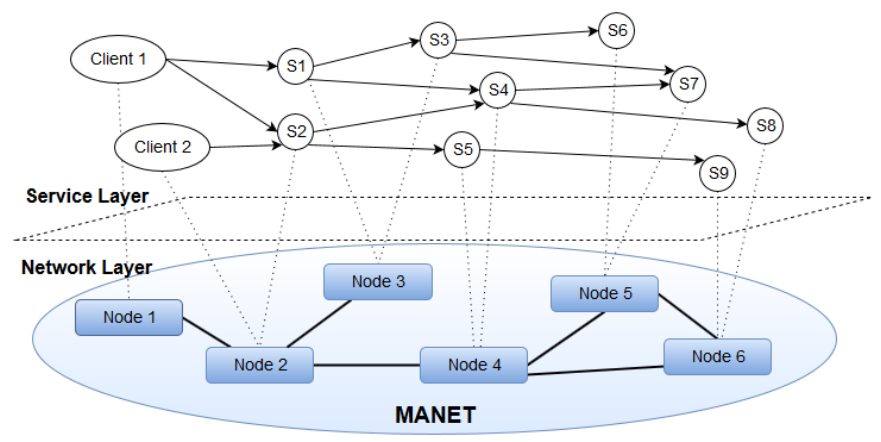

Fig. 1. Hosting service-based systems on MANETs.

ysis data are severely limited, and many of the sources of faults are by their nature transient (e.g., a mobile host that temporarily moves out of radio range).

Further complicating the situation is that faults at the network level may not manifest themselves as failures at the service level, since some of those faults may occur outside of the time during which the relevant services are communicating, or involve communication outside of the relevant faulty conversation. Moreover, service-based systems are designed to mask certain kinds of faults through mechanisms for dynamic service discovery and (re)binding. Therefore, a fault localization method suitable for MANET-hosted servicebased systems must be adept at sifting through noisy data.

We have designed a fault localization method that makes use of two kinds of basic information: (1) service dependence data and (2) network- and service-level fault symptoms. The dependence data and fault symptoms are collected by monitors positioned at network hosts. In the case of dependence data, the monitors observe message traffic carrying service requests and responses, while in the case of fault symptoms, the monitors extract data from local logs. Dependence data are harvested on demand from the monitors and used to 
form a service dependence graph rooted at the client that initiated the conversation and reported the failure [23], [25]. Similarly, fault symptoms are harvested from the monitors. The dependence graph and symptoms are then used to carry out a fault analysis based on a fault propagation pattern, which indicates how faults can be propagated through the system from root causes to clients. The result of the analysis is a fault propagation model that relates possible root causes to the failure reported by the client. Finally, the faults are ranked for their likelihood of causing the failure.

Existing fault localization methods designed for the MANET environment [5], [6], [8], [9], [12], [21], [22], [28] focus on network-level identification of individual faulty hosts and/or the links between them. The methods are limited to low-level observations and measurements of packet flows and host failures, and therefore are blind to the end-to-end context of the service-level conversations affected or unaffected by those faults. Our fault localization method instead makes use of both service- and network-level information. This enables a decoding of the fault propagation through the hosted services via the network. The client is therefore a good entry point for fault localization, tracing failures back to their sources using information that is unavailable from observations at the network level alone.

Clearly, our method is subject to inaccuracies due to effects such as the delay between data collection and data analysis, and inaccuracies in the discovered dependencies representing the system structure. The essence of the present work is to understand how these and other factors impact the accuracy of our method, subject to various tuning and environmental parameters. We focus on the formulation of a basic method that assumes the complete availability of dependence and symptom information for all non-failing hosts in the network. However, in the longer term, but beyond the scope of this paper, we are also studying how incomplete dependence and symptom information can be accommodated in fault localization.

Our evaluation of the method is concerned with its sensitivity to a distinguishing aspect of the MANET environment, namely the faults induced by the dynamism of the network topology. The evaluation is carried out through a series of simulation-based experiments under various scenarios that represent a range of mobile-network dynamics, service dynamics, and critical parameter settings. Because no benchmarks yet exist for MANET-hosted service-based systems, we develop synthetic data to explore the space of independent variables. The dependent variable in these experiments is the mean position of the correct fault in the ranked list of candidates.

To summarize, this paper makes the following specific contributions:

- a method to synergistically analyze fault symptom data gathered at both the service and network levels;

- a fault propagation model based on propagation patterns devised specifically for the operational environment;

- timing- and Bayesian-network-based root-cause ranking techniques; and

- an extensive experimental analysis.

We next review related work. In Section 3 we present background and assumptions we make about fault localization.
In Section 4 we describe in detail our method, including its underlying fault propagation model and ranking algorithms. We then present our experimental evaluation of the method in Sections 5 and 6, and conclude with a summary of our observations and a look at on-going and future work in Section 7.

\section{Related Work}

Although dependability and fault localization have been studied extensively for wired network environments [29], [32], attempts to solve the problem for wireless networks are only more recent. Some of these newer approaches address the issue in the context of stationary, infrastructure-based wireless networks [1], [26], [27], so do not account for the dynamics of MANETs. The few existing approaches to fault localization in MANETs can be generally classified into two broad categories.

In the first category fall those seeking to identify a set of fault-free hosts by comparing the "outputs" of those hosts either to predefined oracles or to the outputs of other hosts [7]. In the basic method, predefined tasks are assigned to network hosts and their outputs are compared to ones defined a priori; a host is considered fault free if the results match. This approach was introduced into MANETs by Chessa and Santi [6]. In their approach, hosts continuously diagnose the status of their immediate neighbors and learn the status of all other hosts in the network via dissemination protocols. Variations and improvements, using different task assignment strategies and/or state dissemination techniques, have also been developed [2], [8], [9]. Because these approaches focus on the identification of fault-free hosts through continuous monitoring and dissemination, they are indirect (and inefficient) methods for fault localization.

In the second, more relevant category are methods seeking to identify individual faulty hosts and links in MANETs. Natu and Sethi [21], [22] use a temporal event-correlation approach with dynamic discovery of the network topology. Their method periodically identifies the end-to-end paths and the states of hosts based on correlations of measurements, identifying faulty hosts and links through active probing of paths. Fecko and Steinder [12] use a combinatorial approach and variance analysis to correlate the occurrences of multiple failures in a network. Their approach is based on probabilistic dependence graphs obtained from expert knowledge of the intended system structure and the history of system faults. Cavalcante and Grajzer [5] propose a form of probabilistic fault propagation model based on Bayesian probability for a specific architectural model of dependable networks [34]. Gupta et al. [13] construct fault signatures for known faults to identify faulty components from symptom observations. Vashist et al. [35] use locally and globally derived symptoms of faults from statistical analysis of network traffic measurements to detect network partitioning as well as faulty links and nodes. Sapello et al. [28] transfer time series data in streams from monitors to analysis nodes to detect individual faulty and noisy links. All these methods are limited by the fact that they focus on identifying the faults at low-level network components (hosts and links), not taking into account the applications actually making use of the MANET. Because 
network-level faults do not necessarily manifest themselves as failures in the application layer and (obviously) vice versa, there is a conceptual disconnect that severely reduces the effectiveness and usefulness of these methods. In contrast, we leverage information available at both levels.

Our method falls into a third category of approaches based on simple graph-theoretical techniques. These approaches use a graph model to describe a set of symptoms that can be observed if a specific fault occurs. The fault localization algorithm then analyzes the model to provide explanations of the observed failures. These approaches are particularly well suited to distributed systems, such as service-based systems, because they allow the complex hierarchical and multi-level structure of the systems to be naturally represented.

We further distinguish between two subcategories of graph-theoretic approaches. The first subcategory uses a dependence graph of the system to represent a set of system components and their dependencies [17], [30]. Although dependence graphs provide a suitable representation of a subject system, existing methods are limited to modeling only a single type of failure per system component. The second subcategory uses a fault propagation model (FPM), a kind of causality graph, to represent various types of events (e.g., symptoms) occurring at a system's components and the cause and effect relationships between those events [14], [18]. Fault localization methods based on an FPM provide a set of candidate root causes explaining the observed symptoms. However, it has been demonstrated that the analysis of a full-blown FPM is an NP-hard problem [4], [17].

In order to manage the complexity of an FPM-based fault localization analysis, existing approaches typically impose limits on the FPM. For example, the FPM may be restricted to a bipartite graph [5], [18], [31]. Other approaches restrict the number or types of faults included in the FPM [4], [18].

Our fault localization method does not impose such arbitrary structural limits on the FPM, which serve to introduce inaccuracies in the analysis. Instead, we construct the FPM from dependencies and symptoms that occur within a restricted period of time relevant to the analyzed fault. This leads to substantial improvements in the effectiveness of fault localization, as we demonstrate in this paper.

\section{BACKGROUND AND ASSUMPTIONS}

In service-based systems, a fault is an exceptional condition occurring in a software (i.e., service) or hardware (i.e., network link or node) component. A fault that occurs during a conversation propagates in a cascade of transitive failures of otherwise fault-free dependent services back to the client that initiated the conversation. Of course, not all faults result in failures if they can be masked. The failures are either exceptions, representing explicit failure notifications in response messages, or timeouts, representing implicit failure notifications caused by response messages not arriving within a specified time period. Typically, the failure does not carry any information about its cause or its source.

The goal of our fault localization method, then, is to uncover the root cause fault of a failure in a conversation. The failure notification received explicitly or implicitly by a client is hence the entry point to our fault localization method. The method makes use of both service- and network-level information to attempt to trace the failure back to its root cause.

When a fault or failure occurs in a system component it manifests as a symptom. To trace the propagation of failures through the system components (i.e., the services, nodes, and links), we must track those symptoms. In order to be minimally intrusive, we restrict ourselves to symptoms that are already recorded in system logs in existing service platforms and network stacks. Of course, some faults and some failures are irrelevant from the point of view of a particular client conversation. For example, the mobility of hosts cause network failures that may or may not disrupt a given conversation. Such symptoms create noise in the data.

The error that is at the heart of a fault (e.g., a software bug, a drained battery, or radio interference) is not significant for the purposes of our analysis method. As long as the root cause fault led to a propagation of failures, the method can be used to reveal the source. However, the method is not designed to address faults that do not cause failed conversations, such as software errors that simply provide incorrect results.

The fault localization method uses a set of distributed monitors, as illustrated in Figure 2. The monitors are deployed in service components (e.g., in a service container) and are responsible for extracting the symptoms of service failures from logs. The monitors provide the symptoms, on demand, to a fault localization element (e.g., located at an operator's station) that runs the fault localization method.

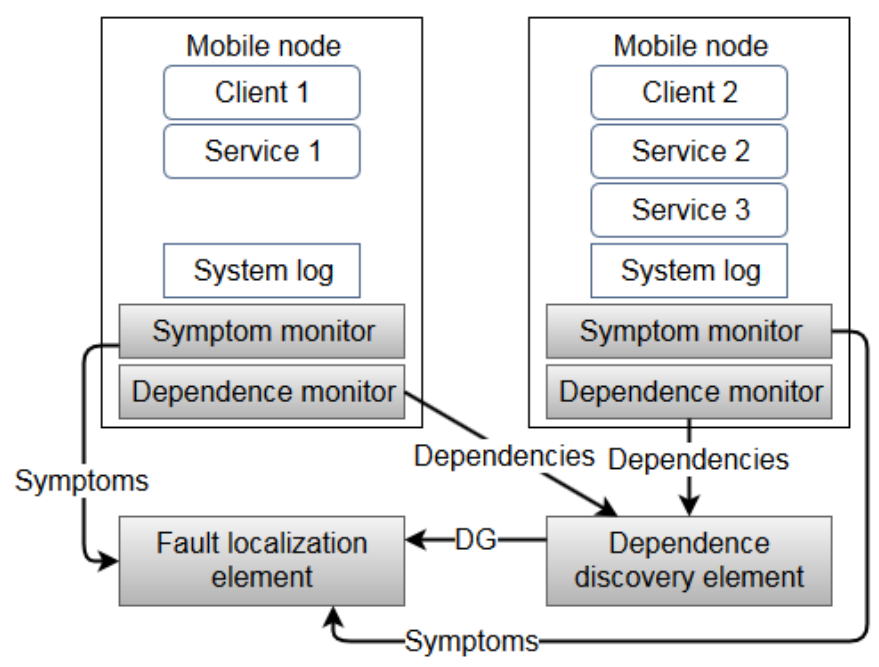

Fig. 2. Monitoring for dependence and symptom data.

An essential input to our method is a dependence graph (DG) that captures the run-time dependencies among services. In service-based systems, a dependence is a relation between services defined by the message flow induced by a client request. (As an edge case, a dependence is also the relation between a client and a service. Without loss of generality, we mainly focus here on relations among services.) When a dependence relation exists between two services S1 and S2, one service is considered the source and the other the target. In general, sources issue requests (i.e., method calls) on targets, thus defining a directionality to the 
dependence. Targets are expected to provide replies (i.e., response messages) back to sources. A DG can be used to represent the full set of dependence relations in the system, or can be restricted to a subset of those relations. For our purposes, we restrict the DG to the dependencies rooted at a particular client and for a particular time period. For example, in Figure 1, the DG of Client 1 consists of all services shown in the network.

A DG can reflect the presence of two kinds of dependencies: inter-dependencies and intra-dependencies. An interdependence is the basic dependence relation that exists between the requester of a service and the direct receiver of that request. An intra-dependence, on the other hand, is a relation between services that indicates which interdependencies emanating from a given service $S$ (i.e., service requests made by S) correspond to which of the individual inter-dependencies incident upon S (i.e., a service request made on S). We need intra-dependencies in situations where not all service requests made on $S$ result in $S$ cascading further requests to all the services on which it depends. Thus, intra-dependencies reflect more detailed insight into the nature of the dependencies between services than do basic inter-dependencies alone, since they are based on observations of how requests flow through a service. In this sense they increase the accuracy of the DG, albeit under the assumption that internal request processing is observable by monitors, which may not always be the case.

Further discussion of dependence graphs is beyond the scope of this paper, suffice it to say that in earlier work we developed a concrete method to obtain probabilistically accurate DGs in MANET-hosted, service-based systems [23]. The dependence discovery method is designed to take account of the peculiarities of that environment, specifically the dynamic nature of service-level binding and host-level mobility, combined with the general lack of resources for tracking dependencies. The method works by building (i.e., "discovering") the DG for a client on demand, which is an approach that is particularly well suited to fault localization because it allows us to obtain the DG for the client that has reported a failed conversation.

Of course, due to the difficult operational environment, the resulting DG can be expected to contain some false dependencies, as well as miss some true dependencies, although the discovery method is designed to minimize the occurrence of such inaccuracies. We must therefore take account of this in the design of the fault localization method. In our experimental evaluation (Section 6) we evaluate the impact of the accuracy of DGs on the performance of our fault localization method.

In the design of our method, we make the following additional assumptions.

A1: The $\log$ entries include information such as a time stamp, an identifier for the relevant service instance, and the type of symptom.

A2: Time stamps are made globally consistent using synchronized clocks. Clock synchronization in MANETs is a well-researched topic, with techniques available to achieve precision of tens or even single microseconds [37]. The smallest time period we use to distinguish time stamps is on the order of several milliseconds, well within this precision.
A3: There is a global timeout parameter shared by all clients and services. The timeout parameter defines the maximum waiting period for responses to requests. This is easily achieved in systems that use a common application platform, such as .NET or J2EE, which have a predefined default value. In systems that use a mix of platforms, explicit enforcement would be required.

A4: The size of messages exchanged between services is small, as typical of SOAP or REST request and response messages, and can be exchanged within the predefined timeout period. Large files (e.g., images and videos) require a different transport mechanism and a different approach to locating faults than that presented here.

\section{Fault Localization}

The previous section introduces the general architecture we envision for recording and gathering the information needed for fault localization. Assuming this information is available to the fault localization element shown in Figure 2, our method for analyzing the information, shown in Figure 3, consists of the following high-level steps:

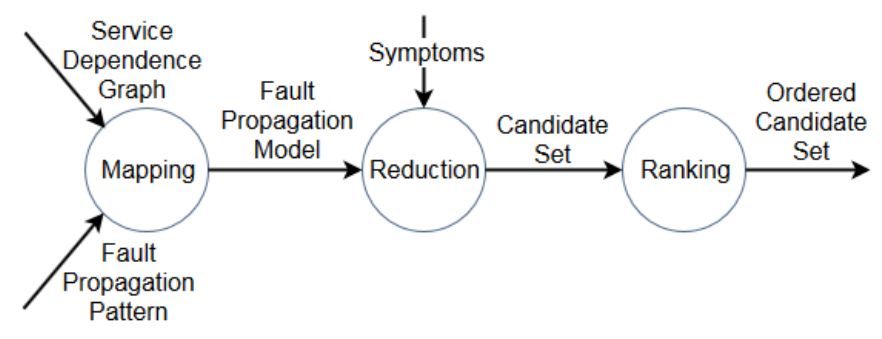

Fig. 3. Fault localization method.

1. Mapping: A fault propagation pattern (Section 4.1), which describes how faults can propagate as failures, is mapped onto the dependence graph associated with a failed conversation. This results in a fault propagation model (Section 4.2), which represents the causality relations between failure events in a system to indicate how specific faults might propagate through the individual services involved in the conversation.

2. Reduction: The fault propagation model is combined with the times that relevant symptoms have occurred (Section 4.2). This assignment allows the method to reconstruct the possible propagation paths for the fault, both in time and in space, and thus to form a set of plausible candidates for the root cause of the failure.

3. Ranking: A ranking (Section 4.3) is applied to the elements of the candidate set based on their likelihood of being the root cause of the failure. We have devised two alternative ranking techniques based on established fault localization methods. The first is a timing approach that ranks hypotheses based on the time difference between possible root causes and the client's fault. The second is a probabilistic method that uses a Bayesian network to infer independent probabilities of individual root-cause hypotheses. We present a comparative evaluation of the effectiveness of these two techniques in Section 6. 


\subsection{Fault propagation pattern (FPP)}

We describe how faults can propagate as failures from one component in a system to another by defining a fault propagation pattern (FPP). More precisely, an FPP is a recursive description of how different kinds of failures can cause further failures, where the first failure in the propagation chain is the failure manifesting the root-cause fault. In service-based systems, the propagation flows upstream in the service dependence structure, from target services back to source services.

To enable a fine-grained analysis using FPPs, we classify the observable failure symptoms-exceptions and timeouts-into failure modes.

A propagated exception (Figure 4a) can be generated as a result of one of the following modes: (1) a send failure (SENDF), which represents a service that is unable to send a message to another service due to a network-level fault; (2) a software failure (SF), which represents any internal software or data fault that throws an exception; and (3) an exception (EX), which is itself generated by a service in response to a failure in a downstream service. Notice that the first two are (manifestations of) actual root causes, while the third is the recursive behavior that leads to propagation.

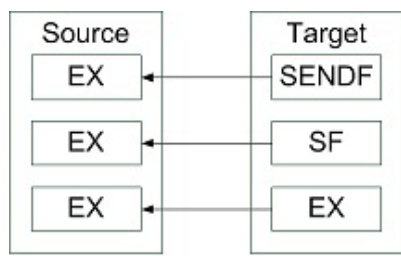

(a)

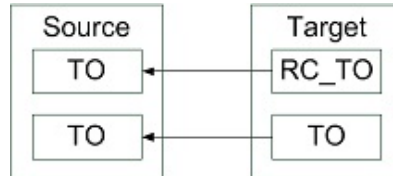

(b)
Fig. 4. Fault propagation pattern refined into modes for (a) exception failures and (b) timeout failures. Faults propagate upstream in the service dependence structure, from targets back to sources.

A propagated timeout can be caused only by some other timeout. This is because the timeout event is an implicit symptom of a fault whose real cause is not directly observable and, hence, not itself recordable as a symptom. Examples include a network host that moves out of communication range or the physical failure of a host. We identify two timeout modes (Figure $4 \mathrm{~b}$ ) that can cause further upstream timeout failures: (1) a root-cause timeout mode ( $\left.R C_{-} T O\right)$, which results implicitly from a network-level fault, and (2) a transitive timeout mode (TO), which results from a timeout occurring in some downstream service.

A subtle and counter-intuitive point: A timeout failure is consistently witnessed by a client before the root-cause RC_TO failure occurs. This is because the client starts its response timeout timer when it initiates the conversation, and all downstream timers in the conversation are also set to this same default value (assumption A3). Thus, the client's timer would actually be the first to expire in the conversation.

\subsection{Fault propagation model (FPM)}

Our technique makes use of a graph, called a fault propagation model (FPM), representing the causality relations between failure events in a system. An FPM is the result of mapping the fault propagation pattern (FPP) described above onto the service dependence graph (DG) of a given client. It is a rooted reverse-directed acyclic graph whose nodes and edges correspond to the client, services, and dependencies of the DG. More precisely, the graph contains a single node at its root to represent the failure mode witnessed by the client, and a set of nodes at its leaves to represent the failure modes of the candidate root causes. The internal nodes represent transitive failure modes. The edges signify possible failure propagation paths, which follow the reverse dependence edges in the DG. Figure 5a shows an example constructed by mapping the exception FPP of Figure 4 a onto the DG of Client 1 in Figure 1.

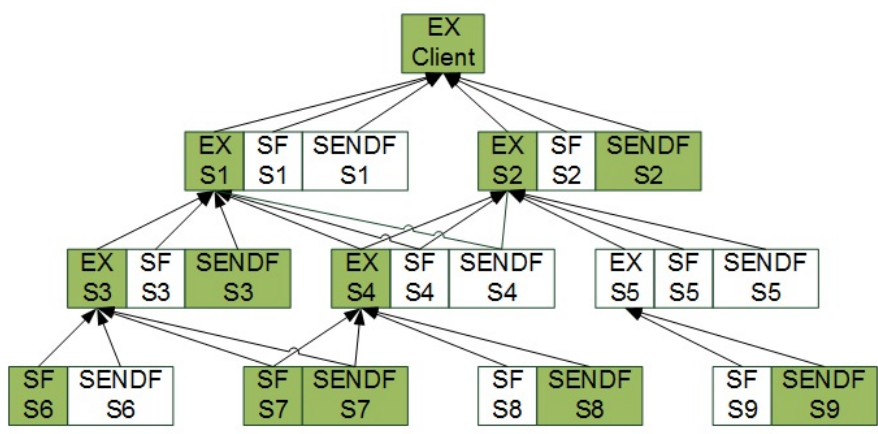

(a)

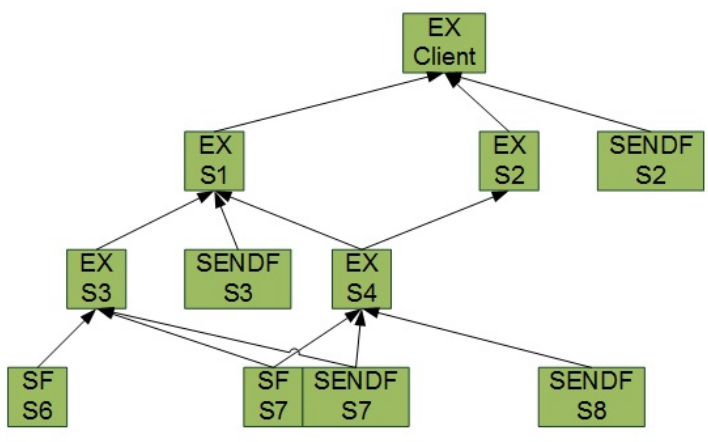

(b)

Fig. 5. Fault propagation model constructed from exception modes of Figure 4a mapped onto dependence graph of Client 1 in Figure 1. The full FPM (a) has highlighted modes by considering actual symptoms recorded in a given time window. It is reduced (b) to candidate root cause failures.

Faults tend to be transient in MANETs and can affect the clients only for a limited period of time. In order to capture this temporal aspect of the problem, an FPM typically maintains information concerning a specific time window, reflecting only the failure symptoms collected by monitors during that period. The time window is a property of the interaction between the application behavior, the network behavior, and the information accessible to monitors. The selection of the time window size is critical in the construction of the FPM. For example, a small time window serves to reduce the size of the FPM, but some critical service interactions and symptoms might be missed. A large time window provides a more complete record of dependencies and symptoms, but might include stale or irrelevant interactions and symptoms (i.e, those belonging to conversations other than the conversation of concern to the analysis). In principle, one needs to consider how long it would take all potential root-cause faults to propagate to 
a client. Ideally, the time period should be long enough to cover the longest such propagation, but no longer, so as not to include considerable noise in the data. We examine this issue carefully in the context of our experimental evaluation in Section 5.3.

Operationally, upon receiving a failure report from a client, the fault localization element builds the FPM with respect to the reported failure. To do so, the dependence discovery element is queried for the DG associated with the failed conversation. The DG is then combined with the FPP and transformed into an FPM for the specific kind of failure, according to the following steps:

1) Every leaf service of the DG is transformed into the root-cause failure modes of the FPP; every intermediate node of the DG is transformed into root-cause and transitive failure modes; and the client node is transformed into a single transitive mode representing the client failure.

2) The edges representing cause-and-effect relations between modes are assigned based on the dependencies in the DG such that every mode of a target service is connected to the respective mode of a source service. The resulting FPM represents all possible root-cause modes with respective propagation paths, given the structure of the DG.

3) The fault localization element contacts the monitors of the services included in the DG/FPM in order to harvest locally aggregated symptom data for the time window of interest. The aggregation applied depends on the kind of failure mode associated with the recorded symptom: for exceptions it is the latest in the time window, while for timeouts it is the earliest. (The justification for these aggregations is given in Section 4.3.) The data are then assigned to the relevant modes of the FPM.

4) The FPM is reduced to a set of candidate root-cause failure modes based on the actual symptoms recorded within the given time window. To do so, any modes (whether transitive or root cause) without associated symptoms are removed from the FPM, as they (probabilistically) could not have either caused or propagated to the client. The FPM is then further reduced to the set of candidate root-cause modes, which is connected to the client failure mode either directly or by propagation paths through the remaining transitive modes. The reduction repeatedly removes all modes that do not have a following (parent) transitive mode. It also removes all transitive modes that do not have any preceding (child) mode.

The resulting FPM contains the set of candidate root-cause failure modes that can reach the client directly or through transitive modes. The FPM also contains the set of transitive modes that are on propagation paths between at least one root-cause mode and the client. For example, the FPM of Figure $5 a$ can be reduced to the FPM of Figure $5 b$ if we assume the following symptoms are recorded in the given time window: services S6 and S7 experienced software failures (SF); services S2, S3, S7, and S8 experienced message send failures (SENDF); and services S1, S2, S3, and S4 received exception messages (EX). The client, which pre- sumably triggered the analysis, also received an exception message (EX).

The method used to select and harvest symptom data is the same as that used to harvest dependence data [25], since the relevant nodes are reflected in the structure of the DG. Hence, the symptom data harvesting mechanism is designed as an extension of the dependence data harvesting mechanism such that the symptom data are transmitted along with ("piggybacked" on) the dependence data. In this way, the amount of network communication can be minimized.

\subsection{Ranking candidate faults}

Given a reduced FPM that consists of possible root-cause failure modes, the next step is to rank the candidate rootcause faults in the order of their likelihood of being the actual root cause of the client failure. We consider two techniques for ranking the candidates: one based on occurrence time and the other based on Bayesian probabilities. We compare the two approaches in our experimental evaluation in Section 6.1.

Timing-based ranking. The timing-based ranking technique follows from an observation: Although conversations might last a long time, failures will propagate relatively quickly from root causes to clients. For instance, intermediate services typically generate exception responses immediately upon receiving an exception response from a downstream service in the conversation, ultimately causing the client to see the exception within a relatively short time after the root cause fault has occurred. Similarly, timeout events in the services involved in a conversation tend to occur within a relatively short period. This is due to the fact that services set their timeout timers when they send service requests, and the forward propagation of those requests through the system is typically much quicker than the time it takes for the conversation as a whole to complete.

Our timing-based algorithm therefore simply ranks the candidate root-cause failures appearing in the reduced FPM in increasing order (from shortest down to longest) in terms of the difference between the time stamp of the client failure symptom and the time of occurrence of the candidate, which is itself determined by the time stamp of:

- the latest symptom of the failure, in the case of an exception, and

- the earliest symptom of the failure, in the case of a timeout.

This aggregation ensures that the ranking favors the candidate that occurs closest in time to the failure witnessed by the client.

Bayesian-network-based ranking. Our second technique assigns the ranks to the candidates in terms of the probability that the candidate is the root cause. The probabilities are inferred on the Bayesian network (BNet) constructed from the FPM. We use a multi-level BNet model, where each node of the graph is a binary-valued random variable that describes the state of a single FPM mode. ${ }^{1}$ The BNet is an isomorphic transformation of the reduced FPM. However, the direction of the BNet edges is reversed from FPM edges

1. http://code.google.com/p/bnt/ 
because in the BNet we measure the probability of each candidate node to be a root cause, given the evidence that the client witnessed a failure. Thus, we measure the probability of propagation in reverse order.

For the failure propagation probabilities in the BNet, we use the noisy-OR gate distribution model of the conditional probability distribution (CPD), due to its computational and space efficiency [15]. The CPDs are calculated based on two assumptions concerning fault propagation. First, given that the reduced FPM only provides the relevant subset of candidate root causes, the probability of the antecedent mode to propagate to a dependent mode is equal for all transitions. Second, the probability of a candidate to be the root cause decreases with the increasing number of transitions through which the fault propagates to the client.

Therefore, we calculate the CPD of a node in the BNet such that the conditional probability of a parent node in the CPD, given the occurrence of the mode in a child node (i.e., the probability of propagation from parent to child node), is equal to the proportional fraction that the parent represents in the set of all parents of the particular child node in the FPM. For example, a child node with two parents has a CPD of $50 \%$ propagation probability of/from each parent. Furthermore, in order to reflect the decreasing effect of each transition on the probability of the mode to be the root cause, the conditional probability of each parent is modified with the constant 0.99 . This constant is sufficient to distinguish between the candidate modes at different levels of the FPM, yet small enough not to interfere with the parent probabilities.

For the inference of the posterior probability of the root cause modes, we use the junction-tree algorithm [33], [36], which is an exact inference algorithm suitable for smallto-medium sized, multi-level directed acyclic graph models such as those we expect in our analysis context.

\section{Experimental Methodology and Setup}

The evaluation of our fault localization method is based on a simulation framework for service-based systems operated in MANETs [24]. As shown in Figure 6, the framework first generates message and fault traces according to various simulation parameters and scenario configurations. These traces are stored in a database to hold the results of the simulation runs. The traces are used to produce dependence graphs and FPMs; these analyses are prototyped as queries over the trace database. We also derive the "ground truth" to evaluate the accuracy of our method. Finally, we compute rankings of candidate root causes using the two techniques described in the previous section.

The simulation engine is built on top of the discreteevent network simulator NS- $3{ }^{2}$ which provides a comprehensive network simulation using detailed implementations of low-level network protocols. However, the highest abstraction provided by NS-3 is that of sockets and network packets. We extended NS-3 with higher-level abstractions for simulating service entities and their interactions. We did this by first encapsulating the socket layer into a messaging layer that provides the abstraction of service-level messages.

2. http://www.nsnam.org/

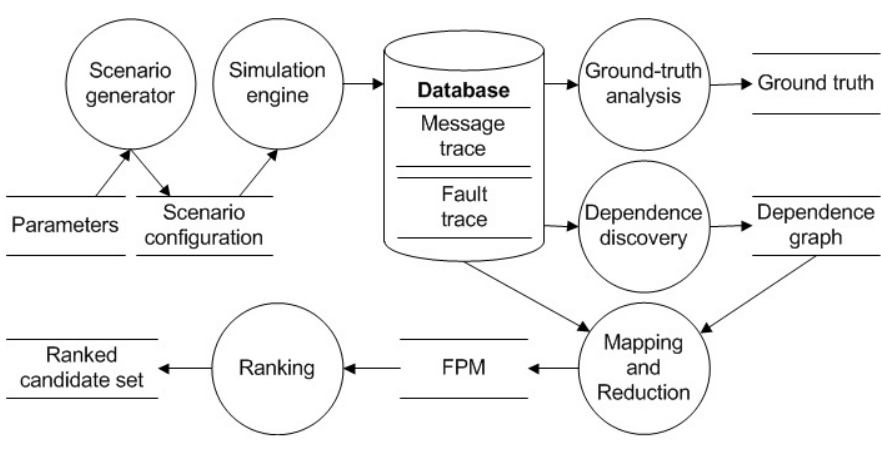

Fig. 6. Experimental framework.

The messaging layer itself is then encapsulated into a service layer that provides abstractions for services, clients, and their service-oriented interactions. Finally, we provide a means to configure and run the extended simulation.

The simulation makes use of various network-layer and service-layer parameters, as we explain below. We collect our results from 30 minutes of simulated execution time after excluding a 30 second warm-up period. Each combination of parameters results in thousands of conversations occurring during the simulation, among which the failed conversations constitute the statistical samples of our analysis subject to the randomness in the service request times, host mobility, wireless signal fading, and the like.

\subsection{Parameters}

To model the operational conditions of a service-based system deployed in a MANET environment, we posit a realistic configuration of network- and service-level parameters.

Table 1 summarizes the basic network-layer parameters used in our simulations. These are standard settings used widely in the networking community and already embodied in the NS-3 simulator. Specifically, we use the log distance model with path-loss exponent 3 for wireless signal propagation, reproducing a network operated in an urban area [10]. We set the spatial mobility bounds to a 75 meter square, which is a limitation imposed by the chosen WiFi standard, as larger regions induce long-term network partitioning. Another important parameter is the mobility speed of the mobile hosts. For most of the experiments we set the mobility speed of all nodes to $10 \mathrm{~m} / \mathrm{s}$. The significance of high mobility is that the hosts are frequently disconnected from each other and, as a result, the message exchanges between the services fail frequently, causing message send failures (SENDF) and timeout failures (RC_TO) in the context of our fault models.

Table 2 summarizes the basic service-level parameters used in our simulations. The message sizes and timeouts are derived from typical values found in many SOA and Web Services implementations. In particular, following assumption A4 (Section 3), the message size is selected uniformly at random from an interval of 500 to 1500 bytes representing simple SOAP or REST request and response messages [3]. The default timeout of response messages varies across application frameworks and can even be adjusted as needed. In our experiments we chose 60 seconds as a single representative value; choosing any other value would not 


\begin{tabular}{|l|r|}
\hline Number of nodes & 50 \\
Spatial bounds & $75 \mathrm{~m} \times 75 \mathrm{~m}$ \\
Mobility speed & $10 \mathrm{~m} / \mathrm{s}$ \\
Mobility model & RandomDirection2dMobility \\
Propagation delay model & ConstantSpeedPropagationDelay \\
Propagation loss model & LogDistancePropagationLoss $/ \alpha 3$ \\
WiFi standard & $80211 \mathrm{~b}$ \\
WiFi rate & $11 \mathrm{Mbps}$ \\
Routing protocol & OLSR and Ipv4StaticRouting \\
Protocol stack & UDP/IPv4 \\
\hline
\end{tabular}

TABLE 1

Network-layer parameters.

\begin{tabular}{|l|r|}
\hline Number of clients & 50 (one per node) \\
Number of services & 30 \\
Invokable methods per service & 2 \\
Workload (client request rate) & $10 \mathrm{~s}-80 \mathrm{~s}$ \\
Message size & $500-1500$ bytes \\
Response timeout & $60 \mathrm{~s}$ \\
Inter-service connectivity probability (Low) & 0.0125 \\
Inter-service connectivity probability (High) & 0.05 \\
\hline \multicolumn{2}{c}{ TABLE 2 } \\
\multicolumn{2}{|c}{ Service-layer parameters. }
\end{tabular}

\begin{tabular}{|l|r|r|}
\multicolumn{1}{c}{ Low } & \multicolumn{1}{c|}{ High } \\
\hline Services in dependence graph (avg.) & 2.03 & 7.9 \\
Services in dependence graph (std. dev.) & 1.2 & 3.63 \\
Number of conversations in scenario & 8541 & 6810 \\
Ratio of failed conversations & $5.01 \%$ & $20.88 \%$ \\
Proportion of SF root causes & $16.6 \%$ & $16.0 \%$ \\
Proportion of SENDF root causes & $75.7 \%$ & $76.8 \%$ \\
$\begin{array}{l}\text { Proportion of TO root causes } \\
\text { Root-cause symptoms in time window } \\
\quad \text { per client failure EX (avg.) }\end{array}$ & $7.7 \%$ & $7.2 \%$ \\
$\begin{array}{l}\text { Transitive symptoms in time window } \\
\text { per client failure EX (avg.) }\end{array}$ & 34.88 & 102.66 \\
$\begin{array}{l}\text { Services failed with root-cause in time } \\
\text { window per client EX failure (avg.) }\end{array}$ & 13.41 & 125.69 \\
$\begin{array}{l}\text { Symptoms in time window } \\
\text { per client TO failure (avg.) }\end{array}$ & 4.67 & 19.16 \\
$\begin{array}{l}\text { Services failed with root-cause in time } \\
\text { window per client TO failure (avg.) }\end{array}$ & 2.8 & 12.81 \\
\hline \multicolumn{2}{|c|}{ TABLE 3 } & 10.22 \\
\hline
\end{tabular}

TABLE 3

Dependencies and faults induced by connectivity.

impact the accuracy of the fault localization method, but merely shift the time at which the timeout faults occur. The workload (i.e., the rate at which clients issue service requests) is selected uniformly at random from an interval of 10 to 80 seconds, representing a good mix of frequent and infrequent client-to-service interactions. Note that the number of methods in each service is not significant from a simulation point of view, as long as we have at least two methods available so that we can examine the impact of dependence information accuracy on fault localization.

We consider systems exhibiting a two-tiered topology, where the first consists of the connections between the clients and a set of "front end" services, while the second consists of the connections between the services themselves. We use 50 clients, five front-end services, and 25 "back end" services. When starting a conversation, each client invokes a method selected uniformly at random from all methods provided by the five front-end services. We experimented with other topologies, including a single-tier topology in which there are no designated front-end services, and with different numbers of clients and services, but found that the results were consistent.

Of particular importance is the degree of connectivity among the services. Table 2 shows how we configure the simulations to capture two different connectivity scenarios, denoted as "Low", and "High". The connectivity degree is induced by the probability that a method in one service invokes a method in another service; the higher the probability, the denser the interconnection topology.

\subsection{Symptoms exhibited by the simulations}

In addition to the network-level faults caused by mobility and communication errors, we also cause two kinds of service-level faults: In the first kind, the service fails to generate proper responses to the service requests and instead generates exceptions, while in the second, the service itself fails due to, for example, a host failure, and so does not generate any response. In our context, a fault of the first kind corresponds to the root-cause of an EX failure, while the second results in the root-cause of a TO failure. We use a service fault rate of $0.5 \%$, which means 5 out of every 1000 service requests fail. We configure the simulations such that $90 \%$ constitute exception root-cause faults (SENDF or SF) and $10 \%$ timeout root-cause faults (RC_TO).

Table 3 summarizes the failure symptoms observed in our simulations, where the columns "Low" and "High" represent sparse and dense service connectivity scenarios, respectively. For instance, the low-connectivity configuration (combined with $0.5 \%$ service fault rate and $10 \mathrm{~m} / \mathrm{s}$ mobility speed) results in 8541 conversations. The average number of services in the dependence graph of each conversation is larger in highly connected service configurations compared to that of lower connectivity configurations (approximately 8 versus 2). A high standard deviation in this case indicates a good mix of different kinds of conversations. The ratio of the failed conversations is about $21 \%$ of all conversations in the high-connectivity scenario, compared to about $5 \%$ in the low connectivity scenario. Of all root-cause faults, SENDFs constitute about $76 \%$. These are caused by faulty network links, rendering services unable to successfully send request messages to other services. In comparison, timeout faults occupy a small portion of only about $7 \%$. Of the remaining root-cause faults, about $16 \%$ are caused by the injected software faults.

A secondary effect of the ratio of failed conversations is the number of faults occurring in a given time period. For example, as shown in Table 3, the average number of faults occurring in all services during the relevant time window prior to the client exception is, in the low-connectivity scenario, about 35 root-cause faults and 13-14 transitive failures. In the high connectivity scenario, this number increases to nearly 103 root-cause faults and 126 transitive failures.

The average number of failing services during the relevant time window (i.e., the services that experience at least one fault or failure from all overlapping conversations within the time window) prior to the client exception is nearly 5 root-cause faults in the low-connectivity scenario and about 19 in the high-connectivity scenario. Due to the lower rate of timeout faults, the average number of faults occurring in all services during the relevant time window sub- 
sequent to the client timeout is, in the low-connectivity scenario, nearly 3 timeout faults, and in the high-connectivity scenario this number increases to nearly 13 . The average number of failed services during the relevant time window subsequent to the client timeout is about 2 root-cause faults in the low-connectivity scenario and 10 in the highconnectivity scenario.

An increasing number of faults occuring during the time window increases the number of irrelevant symptoms witnessed during a given conversation. As explained in Section 4, irrelevant symptoms can be reduced by making use of the dependence information captured in a DG. However, with an increase in connectivity comes an increase in the size of the DG. Thus, an increase in connectivity has a doubly negative impact on fault localization, causing an increased fault rate as well as an increased portion of the whole system from which the faults are drawn into the analysis. In Section 6.3 we report results in which we vary the connectivity parameter.

The impact of having more services involved in a conversation is the increasing time it takes for faults to propagate from root causes to clients. This is borne out by Figure 7, which gives the cumulative distributions of the time between the occurrence of a root-cause fault and when a client witnesses a failure. For example, $92 \%$ of the exception rootcause faults in the low-connectivity scenario propagate in under 0.03 seconds, whereas only about $50 \%$ do so in the high-connectivity scenario.

The ratio of fault types in a scenario is an outcome of several system properties, such as the behavior of the network, configuration of the services, and the characteristics of message exchanges. When a link between services is broken, a request message will fail to be sent and the resulting fault will propagate as an exception back to the client. However, in some cases, the quality of the link deteriorates only after the request was sent and so it is the response message that fails to arrive back, causing instead a timeout. Aside from these network-induced faults, services may fail due to software or data problems. In order to simulate this behavior, we inject faults into the services such that every service invocation has some probability to fail.

A service can fail and respond with an exception message, which is a common reaction to service faults handled either by the service itself or by the host environment of the service. However, in some cases the service (or its host) may fail "silently". This is a particularly common fault in MANETs, where nodes have limited reliability (due, for example, to limited battery life). We model this kind of faulty behavior in such a way that $10 \%$ of service faults do not produce response messages and, thus, cause timeout root-cause faults.

Overall, in the scenarios we present, more than $92 \%$ of network and service faults cause clients to fail with an exception. We have experimented with various ratios of the SENDF and SF root-cause faults making up this percentage, but find that those ratios have little to no impact on accuracy of our method. Timeout root-cause faults represent only about $7 \%$ of all client failures in our scenarios. This is a significant observation because the identification of timeout root-cause faults is less accurate (due to root-cause and transitive symptoms being indistinguishable). However, be-

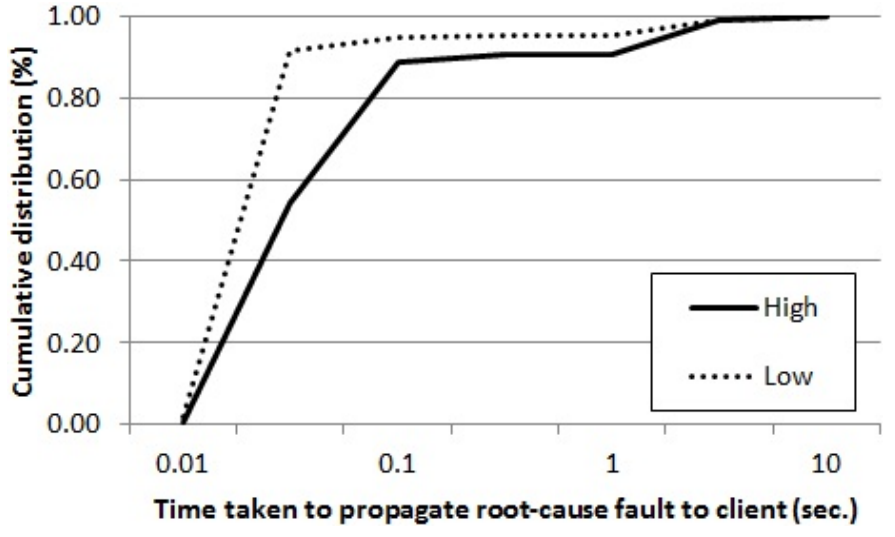

(a)

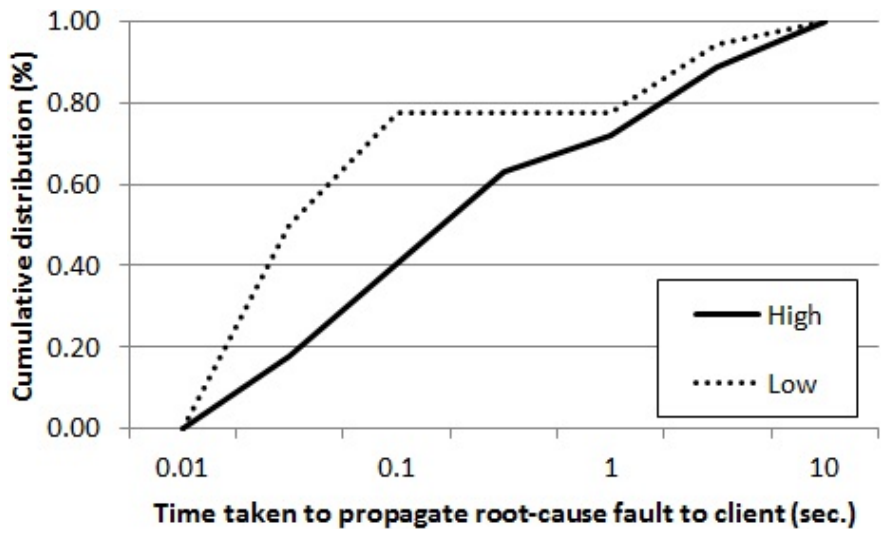

(b)

Fig. 7. Cumulative distribution of time between occurrence of root-cause exception (a) and timeout (b) faults, and when clients witness failures.

cause the fraction of timeouts is relatively small, it has only a small negative impact on the overall accuracy of fault localization.

\subsection{Selection of time window size}

As mentioned in Section 4.2, the time window is an important tuning parameter for our method, since it affects which failure symptoms are included in the FPM. When selecting the size of the time window, one needs to consider how long it would take for root-cause faults to propagate to clients. A naïve approach would be to set the value equal to the longest duration that a conversation can last, which in turn is limited by the service response timeout parameter, since any failure in the system should occur within this period. Following standard . $\mathrm{NET}^{3}$ and similar implementations, the timeout parameter is set to 60 seconds in our experiments. In practice, however, the fault propagation time can be much shorter than the duration of the entire conversation and, hence, the time window size also needs to be selected accordingly.

Referring again to Figure 7, we can see from the distributions that, regardless of the kind of failure and service connectivity, the time difference between the failure seen by the client and the root-cause fault is fairly small: in almost all cases, the faults are propagated within a few seconds. This

3. http://msdn.microsoft.com/en-us/library/System.ServiceModel. Channels.Binding_properties.aspx 
suggests that the time window size needs to be set much smaller than the maximum duration for the conversation so as not to include too many irrelevant symptoms in the analysis. In the results below, we generally use a 6-second time window for this purpose. Note, however, that this value is chosen based on empirical results, and hence should be considered close to ideal. To reflect cases when such an empirical basis is not available, results with a 60-second time window are also shown whenever applicable.

\section{Evaluation}

In our evaluation, the questions of interest center mainly on the accuracy of the resulting candidate set, specifically the ranking of root-cause fault candidates. A good result, of course, would be that our method consistently ranks the correct root-cause candidate at or near the top. We therefore (1) compare the two ranking algorithms, timing and BNet, in terms of the position at which they place the correct root-cause fault; (2) examine the effects of DG accuracymore precisely, the ratio of false positives-used in the construction of an FPM; (3) investigate the impact of service connectivity, which represents the complexity of a servicebased system; and (4) evaluate the sensitivity of the ranking algorithms to a range of service fault rates.

\subsection{Comparison of ranking algorithms}

We first compare the accuracy of the two ranking algorithms. To isolate their effect from those of other parameters, we show the results for high service connectivity with $0.5 \%$ service fault rates, using 100\% accurate dependence graphs (referred to as "ground truth" in Figure 6), which contain exactly the services involved in each conversation.

Accuracy is measured in terms of the ranking position of the correct root-cause fault for each failed conversation in the experiment. The results are given in Figure 8, which shows the cumulative distribution of those positions and their standard deviations. For purposes of comparison, we include the results for the BNet-based algorithm under both 6- and 60-second time windows; the results for the timing-based algorithm are virtually the same for both time window sizes. Notice that the average sizes of the candidate sets under the 6-second time window are 1.5 for exceptions and 2.6 for timeouts, and 5 and 3.5, respectively, under the 60-second window.

We can see that the timing-based method is very effective for exception failures, resulting in more than $90 \%$ of correct root causes placed in the first position of the ranking, under both 6-second and 60-second time windows. Compare this with an ordering of candidates by random guess, which would do so only for $66 \%$ and $20 \%$ under 6 - and 60 -second time windows, respectively. This good result is due to the fact that exception failures are propagated through a quick succession of explicit messages, which makes a ranking based on the time proximity particularly suitable.

On the other hand, the BNet-based algorithm is more effective in ranking the root causes of timeout failures. Under a 6-second time window size, the BNet algorithm places the correct root causes in the first position for more than $95 \%$ of the failures, and for nearly $60 \%$ under 60 -second

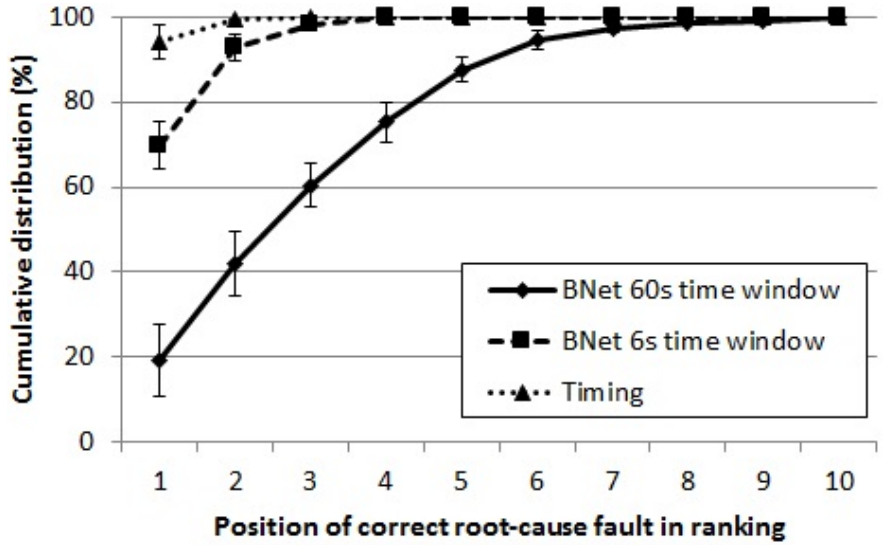

(a)

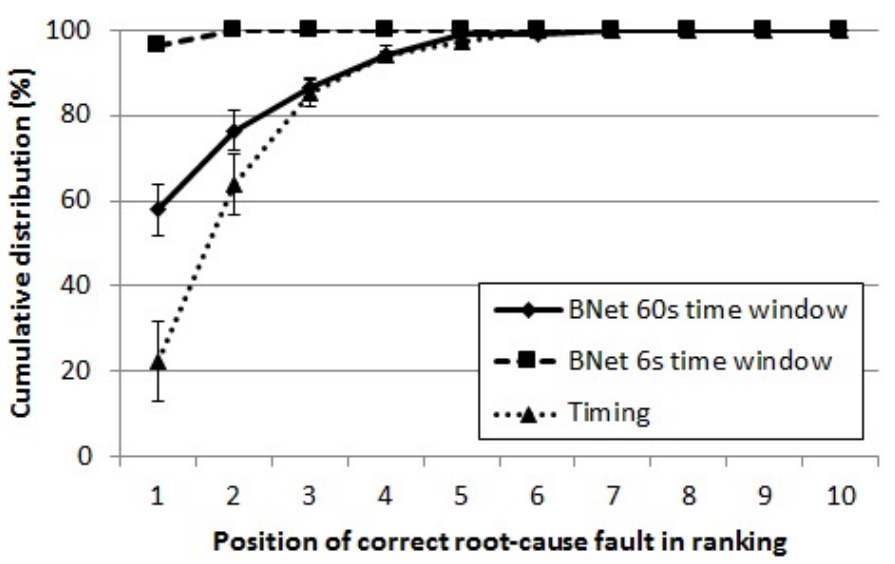

(b)

Fig. 8. Accuracy of ranking algorithms for exception (a) and timeout (b) failures in the high-connectivity scenario. The FPM is constructed from the ground-truth dependence graph.

time window. Compare this again with a random ordering, which would achieve the same only for $38 \%$ and $28 \%$ of the cases under the two time window sizes, respectively. The accuracy of the timing-based algorithm is not good for timeout failures.

The results also show the importance of the time window size for the BNet algorithm. Using the extreme case of 60 seconds, the algorithm produces relatively poor rankings. This is because the data include a substantial amount of noise in the form of irrelevant symptoms. In fact, the candidate sets are much larger, with on average 5 exception and 3.5 timeout candidates, about 3.3 and 1.4 times more than for a 6-second time window in these experiments. Nevertheless, BNet-ranking performs better by placing the correct root-cause at the first position for about $60 \%$ of timeouts; compare this with the results for a random guess, which would do so only for $28 \%$ out of 3.5 candidates.

\subsection{Impact of dependence graph accuracy}

We next investigate the sensitivity of our method to the accuracy of the DG rooted at a client. Recall that the DG is created using a run-time discovery facility that produces probabilistically correct results. Its accuracy can be measured in terms of any extraneous dependencies included in the graph. An extra dependence is a false positive (FP) 


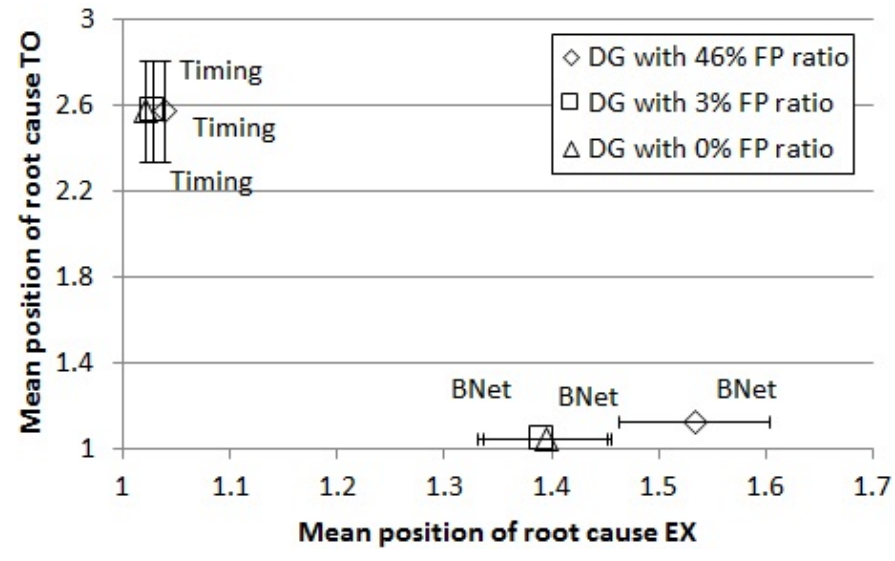

Fig. 9. Impact of dependence graph false positive ratio on mean ranking position of correct root-cause fault. A ratio of $0 \%$ represents the ground truth.

drawn from some other, irrelevant conversation. The ratio of false positives to true positives (the FP ratio) is influenced by various factors, such as mobility speed, service workload, and most importantly the technique used for dependence discovery.

Here we make use of both inter- and intra-dependence information [23]. Recall that to discover inter-dependencies we make use of observations of messages exchanged between services, while to discover intra-dependencies we combine inter-dependence information with observations of requestprocessing flowing through services, thereby increasing the accuracy of the DG (Section 3). They are represented here, respectively, by the FP ratios $46 \%$ and $3 \%$ in our experiments. We also include in our experiments the groundtruth DG that contains no false positives. Again, we show results for the high connectivity scenario and 6-second time window.

Figure 9 shows the impact of the FP ratio on the accuracy of our method under a 6 -second time window. The average ranking positions of correct root causes for exception and timeout failures are plotted against each other and shown for the two ranking algorithms. Consistent with the results in Section 6.1, the algorithms behave differently for the two kinds of faults. Notice that the timing-based algorithm is essentially insensitive to the accuracy of the DG, which reinforces our conclusion that its effectiveness at localizing exception failures is dominated by the time proximity of the failure occurrence. The BNet algorithm also exhibits only some small sensitivity to the DG's accuracy.

\subsection{Impact of service connectivity}

We now turn to the impact of service connectivity (i.e., system complexity) on the accuracy of our method. It is important to investigate this factor because it influences many other contributing factors, such as the size and false positive ratios of dependence graphs, the duration of conversations (and, consequently, the amount of overlap between separate conversations), and the overall failure rate exhibited by the system. We hypothesize that the accuracy of the fault localization method will suffer under highly connected services, since the combined effect of the increases in the above factors should have a negative impact on the ranking position.

We present our results in Figure 10, where we compare the low and high-connectivity scenarios using the same sort of plot as in Section 6.2. These results confirm our hypothesis: in the low-connectivity scenario, our method achieves significantly better accuracy for both ranking algorithms than in the high-connectivity scenario. The better result for low connectivity is due to the relatively small number of services involved in each conversation (on average, 2.03 versus 7.9), the relatively low degree of overlap between conversations, the relatively low dependence graph FP ratio (we use an intra-dependence-based DG with $0.2 \%$ ratio versus $3 \%)$, and a lower inherent failure rate $(5.01 \%$ versus $20.88 \%$ ). The reverse is true for the high-connectivity scenario.

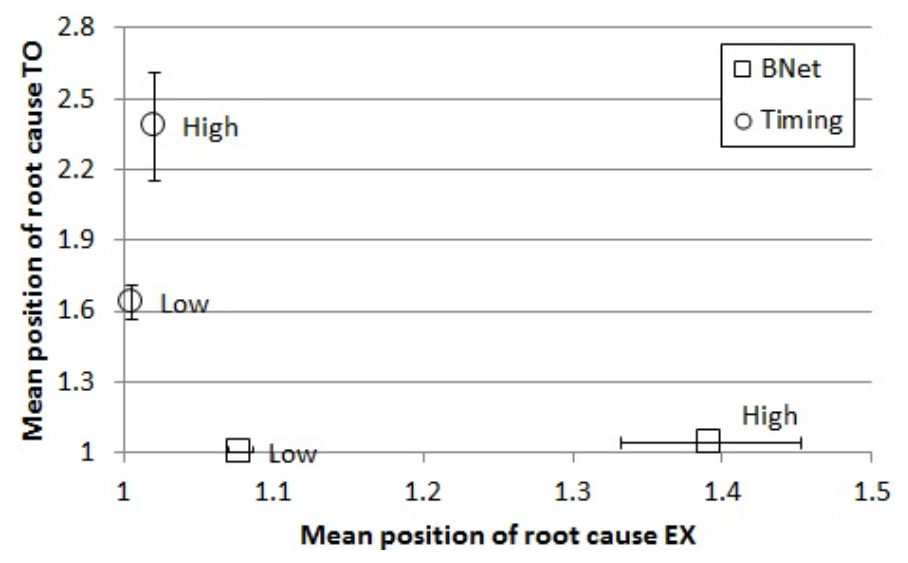

Fig. 10. Impact of service connectivity on mean ranking position of correct root-cause fault.

\subsection{Impact of service fault rate}

In our final experiment, we investigate the accuracy of our method under a range of service-level (software) fault rates. Recall that we configure the simulations so that when a client experiences a failure, $90 \%$ of the time the root-cause is an exception and $10 \%$ of the time the root-cause is a timeout. We expect that an increasing fault rate should be negatively correlated with the accuracy of locating both types of faults.

The results are shown in Figure 11 for both ranking algorithms under the high-connectivity scenario and a 6second time window. The service fault rate is varied in the $x$-axis from $0 \%$ to $2 \%$. For each rate, we also give the total number of failed conversations. Notice that even with a $0 \%$ service fault rate, the scenario reflects failed conversations caused by network faults during the entire simulation.

The results demonstrate that timing-based ranking is fairly robust in a wide range of service fault rates, maintaining consistently high accuracy even under high fault rates. Accuracy of BNet-based ranking deteriorates, however, as the service fault rate increases. This is because the BNet algorithm is sensitive to the number of possible root causes in the FPM, which increases not only due to inclusion of irrelevant dependencies (recall Figure 9), but also due to the increase in the fault rate, causing some irrelevant failures from overlapping conversations to be included in 


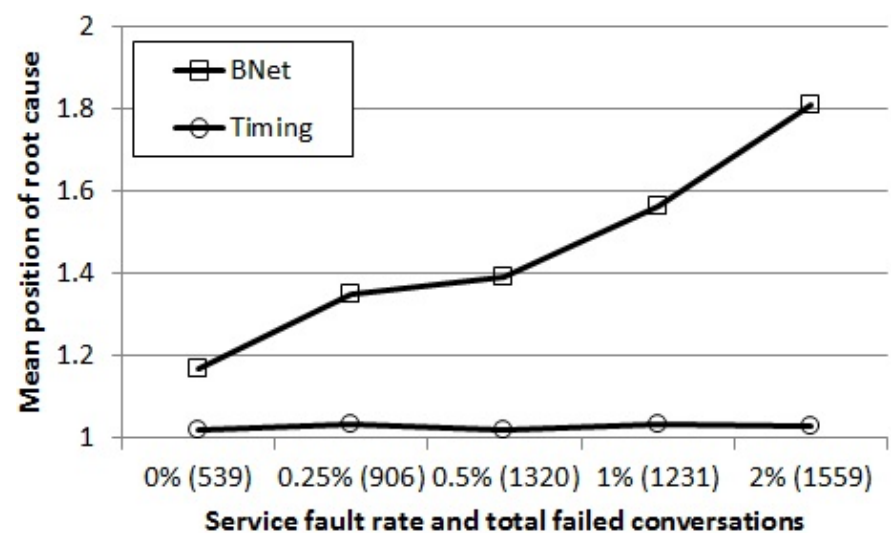

(a)

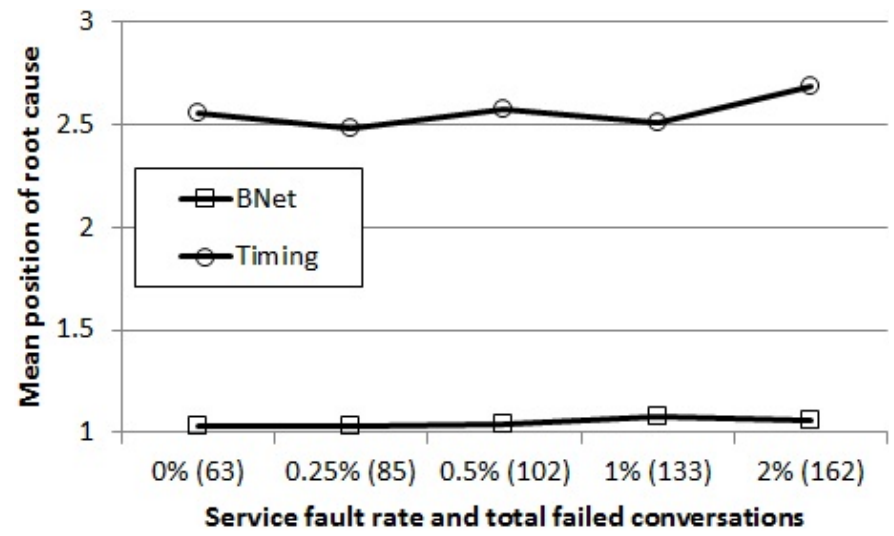

(b)

Fig. 11. Impact of service fault rate on mean position of correct rootcause exception (a) and timeout (b) faults. Both the fault rate and the total number of failed conversations for each rate are shown.

the FPM. The timing-based ranking deteriorates as well, but at a significantly lower rate, since the effect of the lower proportion of timeout root-cause faults, $10 \%$, is minimal.

\subsection{Discussion of results}

The experimental results above demonstrate the effectiveness of our fault localization method in ranking the correct root causes of failed service conversations. In particular, the timing-based ranking is shown to be very effective in localizing the failures caused by service exceptions, while the Bayesian-based ranking is more effective in the case of timeout failures. This suggests these two algorithms can be employed synergistically according to the kind the failure reported by a client.

Our experiments involve a variety of scenarios with diverse operational characteristics for both the network and service-based system. The goal of the experiments is to show the combined impact of these characteristics on the accuracy of our fault localization method. The structure of the service-based system is captured by the dependence discovery method and presented through the DG to the fault localization method. Thus, the DG accuracy and service connectivity are the two main variables through which the operational characteristics impact the accuracy of the method. We have experimented with additional network topologies and system configurations beyond those presented here, and found the results to be consistent.
The results also show the importance of the proper selection of the time window size, where a value much smaller than the longest duration of the conversations is shown to provide good outcomes, reflecting a relatively quick propagation of the failures in the system compared to the time it takes to complete the conversations. Moreover, the method is shown to perform consistently in a wide range of rates at which service faults occur in the system.

An important consideration in the MANET environment is the requirement for data storage and transfer. The fault localization method does not store any data on its own, since it makes use of existing system logs. On the other hand, the dependence discovery method on which our method is based requires some small amount of data to be stored on the network nodes, well within the capabilities of today's mobile devices [23].

In terms of load on the network, our method requires aggregated data for a desired time window to be sent to a central fault localization element for analysis. The bundle sent by an individual monitor contains data for one service and the status of root-cause and transitive modes relevant to the client's fault (i.e., SF, SENDF and EX for exception faults, and TO for timeout faults). The status of each mode is aggregated into a record containing a Boolean value to represent whether any relevant symptom occurred in the queried time window, together with a timestamp for the latest symptom, which is required for the timing-based ranking. In practice, only a single SOAP message is needed for this. A second SOAP message is needed to transfer the dependence data. According to the SOAP protocol, these messages are each about $0.5 \mathrm{~KB}$. If we consider this in the context of the highconnectivity scenario of our experiments, we involve on average 7.9 services in a conversation, and thus the average amount of data transferred over the network would only be about $7.9 \mathrm{~KB}$ per analysis request $((0.5+0.5) \times 7.9)$. This, however, treats the dependence and fault-symptom data separately; the messages used to transfer the data could be combined, thereby substantially reducing this total.

We now consider how each aspect of our fault localization method contributes to the overall results. To do so, we break down the method into the contributions of its three main elements, each of which is applied in turn to obtain a result: (1) the dependence graph, (2) the fault propagation model, and (3) the ranking algorithm. We start from the total number of candidate root-cause faults observed within the selected time window. We then determine how many are eliminated at each step. We use the high-connectivity scenario with $0.5 \%$ service fault rate and 60 -second time window size. The larger window size allows us to more easily see the reduction in each step; results for the 6-second window size show similar trends.

The results are shown in Figure 12. The height of each bar represents the size of the whole candidate set, that is, the number of failed services within the relevant time window period. As noted above and shown in Table 3, there are 19 and 10 candidate root causes on average for the exception and timeout failures, respectively. (The total number of symptoms observed by the monitors is much higher-102 and 13 failure symptoms for exception and timeout failures, respectively-but as the monitors report only aggregated data, the number of candidates is much 


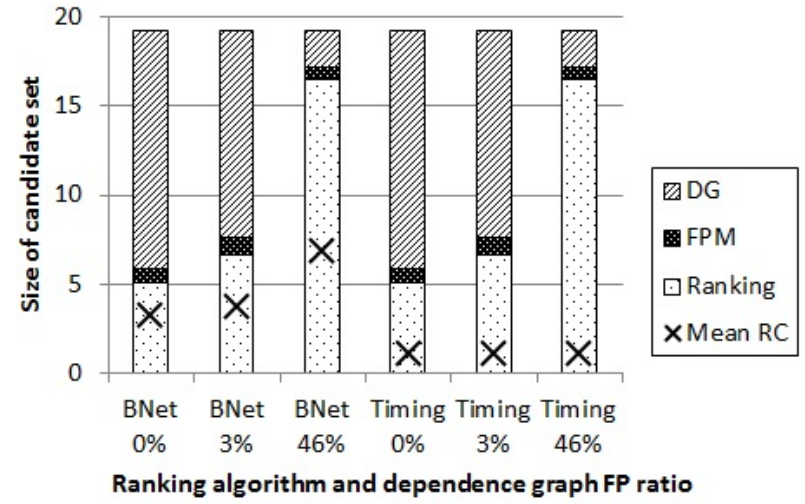

(a)

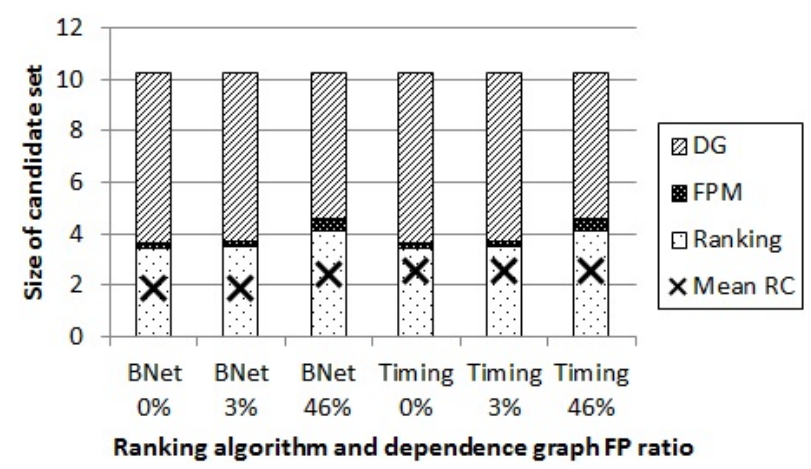

(b)

Fig. 12. Contribution of each method step to the localization of exception (a) and timeout (b) root-cause faults.

smaller.) Each section of the bar represents the number of candidates eliminated by an element of the method: the DG area represents the reduction to those services appearing in the dependence graph rooted at the client that witnessed the failure; the FPM area represents the reduction to those reachable to the client in the FPM; the Ranking area represents the reduction to those including the correct root-cause fault; and the Mean RC represents the final mean position of the correct root-cause fault.

Overall, we can see that each element indeed makes a contribution. The specific contribution differs with the ranking algorithm and accuracy of the dependence graph. The ranking algorithm and DG play the major roles in localizing the root-cause fault of client failures. The effect of DG accuracy is particularly obvious when the $46 \%$ FP ratio is compared against those of $0 \%$ and $3 \%$ for exception failures, suggesting that it is important to have an effective dependence discovery in diagnosing such failures. The timingbased ranking algorithm contributes a significant portion in narrowing down exception failures, even when the DG and FPM are not effective. The BNet-based ranking algorithm is more effective for timeout failures and less so for exception failures. Again, this supports our suggestion to apply the ranking algorithm most appropriate to the reported failure.

\section{Conclusion}

We have presented a run-time fault localization method suitable for service-based systems operated in MANETs. Unlike existing approaches, the method analyses the sources of faults based on failures received by the system clients. The method synergistically analyzes fault symptom data gathered at both the service and network levels, and makes use of a fault propagation model constructed dynamically for the particular client's failure. The FPM contributes significantly to the overall accuracy of the method. The method further provides timing- and Bayesian-network-based algorithms for ranking root-cause candidates.

Through an extensive set of simulation-based experiments, we have evaluated the accuracy of the method in terms of operational factors characteristic of both servicebased systems and MANETs. The method exhibits good behavior when subjected to the stress of a changing underlying network and service topology. Furthermore, the method has minimal data storage, transfer, and computational requirements, scaling well with the number and connectivity of the services involved.

In this paper we have focused on fault localization assuming the availability of complete, albeit noisy, dependence and symptom data. In our current work we are instead developing extensions to dependence discovery and fault localization methods capable of working with incomplete dependence and symptom data.

\section{ACKNOWLEDGMENT}

This research was sponsored by the U.S. Army Research Laboratory and the U.K. Ministry of Defence and was accomplished under Agreement Number W911NF-06-3-0001. The views and conclusions contained in this document are those of the author(s) and should not be interpreted as representing the official policies, either expressed or implied, of the U.S. Army Research Laboratory, the U.S. Government, the U.K. Ministry of Defence or the U.K. Government. The U.S. and U.K. Governments are authorized to reproduce and distribute reprints for Government purposes notwithstanding any copyright notation hereon.

\section{REFERENCES}

[1] A. Adya, P. Bahl, R. Chandra, and L. Qiu. Architecture and techniques for diagnosing faults in IEEE 802.11 infrastructure networks. In Proceedings of the 10th Annual International Conference on Mobile Computing and Networking, pages 30-44, 2004.

[2] N. Aljeri, M. Almulla, and A. Boukerche. An efficient fault detection and diagnosis protocol for vehicular networks. In Proceedings of the Third ACM International Symposium on Design and Analysis of Intelligent Vehicular Networks and Applications, pages 23-30, 2013.

[3] N. Apte, K. Deutsch, and R. Jain. Wireless SOAP: Optimizations for mobile wireless web services. In Special Interest Tracks and Posters of the 14th International Conference on World Wide Web, pages 1178-1179, 2005

[4] A. Bouloutas, S. Calo, and A. Finkel. Alarm correlation and fault identification in communication networks. IEEE Transactions on Communications, 42(234):523-533, 1994.

[5] A. Cavalcante and M. Grajzer. Fault propagation model for ad hoc networks. In Proceedings of the IEEE International Conference on Communications, pages 1-5, June 2011.

[6] S. Chessa and P. Santi. Comparison-based system-level fault diagnosis in ad hoc networks. In Proceedings of the 20th IEEE Symposium on Reliable Distributed Systems, pages 257-266, 2001.

[7] E. P. Duarte, Jr., R. P. Ziwich, and L. C. Albini. A survey of comparison-based system-level diagnosis. ACM Computing Surveys, 43(3):22:1-22:56, Apr. 2011.

[8] M. Elhadef, A. Boukerche, and H. Elkadiki. Diagnosing mobile adhoc networks: Two distributed comparison-based self-diagnosis protocols. In Proceedings of the 4th ACM International Workshop on Mobility Management and Wireless Access, pages 18-27, 2006. 
[9] M. Elhadef, A. Boukerche, and H. Elkadiki. A distributed fault identification protocol for wireless and mobile ad hoc networks. Journal of Parallel and Distributed Computing, 68(3):321-335, Mar. 2008.

[10] I. K. Eltahir. The impact of different radio propagation models for mobile ad hoc networks (MANET) in urban area environment. In Proceedings of the 2nd International Conference on Wireless Broadband and Ultra Wideband Communications, Aug. 2007.

[11] N. Esfahani, E. Yuan, K. R. Canavera, and S. Malek. Inferring software component interaction dependencies for adaptation support. ACM Transactions on Autonomous and Adaptive Systems, 10(4), Feb. 2016.

[12] M. Fecko and M. Steinder. Combinatorial designs in multiple faults localization for battlefield networks. In Proceedings of the IEEE Military Communications Conference, pages 938-942, 2001.

[13] D. Gupta, P. Mohapatra, and C.-N. Chuah. Diagnosing failures in wireless networks using fault signatures. In Proceedings of the IEEE International Conference on Communications, pages 1-5, May 2010.

[14] M. Hasan, B. Sugla, and R. Viswanathan. A conceptual framework for network management event correlation and filtering systems. In Proceedings of the IFIP/IEEE International Symposium on Integrated Network Management, pages 233-246, 1999.

[15] D. Heckerman. A tractable inference algorithm for diagnosing multiple diseases. In Proceedings of the Fifth Annual Conference on Uncertainty in Artificial Intelligence, pages 163-172, 1989.

[16] V. Jalaparti, P. Bodik, S. Kandula, I. Menache, M. Rybalkin, and C. Yan. Speeding up distributed request-response workflows. In Proceedings of the ACM SIGCOMM Conference, pages 219-230, Aug. 2013.

[17] I. Katzela and M. Schwartz. Schemes for fault identification in communication networks. IEEE/ACM Transactions on Networking, 3(6):753-764, Dec. 1995.

[18] S. Klinger, S. Yemini, Y. Yemini, D. Ohsie, and S. Stolfo. A coding approach to event correlation. In Proceedings of the IFIP/IEEE International Symposium on Integrated Network Management, pages 266-277, 1995.

[19] K. Lund, A. Eggen, D. Hadzic, T. Hafsoe, and F. T. Johnsen. Using web services to realize service oriented architecture in military communication networks. IEEE Communications Magazine, 45(10):47-53, 2007.

[20] K. Lund, E. Skjervold, F. T. Johnsen, T. Hafsøe, and A. Eggen. Robust web services in heterogeneous military networks. IEEE Communications Magazine, 48(10):78-83, 2010.

[21] M. Natu and A. Sethi. Adaptive fault localization in mobile ad hoc battlefield networks. In Proceedings of the IEEE Military Communications Conference, pages 814-820, Oct. 2005.

[22] M. Natu and A. S. Sethi. Using temporal correlation for fault localization in dynamically changing networks. International Journal of Network Management, 18(4):301-314, Aug. 2008.

[23] P. Novotny, B. J. Ko, and A. L. Wolf. On-demand discovery of software service dependencies in MANETs. IEEE Transactions on Network and Service Management, 12(2):278-292, June 2015.

[24] P. Novotny and A. L. Wolf. Simulating services-based systems hosted in networks with dynamic topology. Technical Report DTR-2016-2, Department of Computing, Imperial College London, Jan. 2016.

[25] P. Novotny, A. L. Wolf, and B. J. Ko. Discovering service dependencies in mobile ad hoc networks. In Proceedings of the IFIP/IEEE International Symposium on Integrated Network Management, pages 527-533, May 2013.

[26] L. Paradis and Q. Han. A survey of fault management in wireless sensor networks. Journal of Network and Systems Management, 15(2):171-190, June 2007.

[27] L. Qiu, P. Bahl, A. Rao, and L. Zhou. Troubleshooting wireless mesh networks. ACM SIGCOMM Computing Communications Review, 36(5):17-28, Oct. 2006.

[28] A. Sapello, A. Sethi, M. Nodine, and R. Chadha. Application of time series analysis to fault management in MANETs. In Proceedings of the International Conference on Network and Service Management, pages 150-157, Oct 2010.

[29] V. Stantchev and M. Malek. Addressing dependability throughout the SOA life cycle. IEEE Transactions on Services Computing, 4(2):8595, 2011.

[30] M. Steinder and A. Sethi. End-to-end service failure diagnosis using belief networks. In Proceedings of the IEEE/IFIP Network Operations and Management Symposium, pages 375-390, 2002.
[31] M. Steinder and A. S. Sethi. Probabilistic fault diagnosis in communication systems through incremental hypothesis updating. Computer Networks, 45(4):537-562, July 2004.

[32] M. Steinder and A. S. Sethi. A survey of fault localization techniques in computer networks. Science of Computer Programming, 53(2):165-194, 2004

[33] T. A. Stephenson. An introduction to Bayesian network theory and usage. Technical Report IDIAP-RR-03-2000, Dalle Molle Institute for Perceptual Artificial Intelligence, Feb. 2000.

[34] N. Tcholtchev, M. Grajzer, and B. Vidalenc. Towards a unified architecture for resilience, survivability and autonomic faultmanagement for self-managing networks. In Proceedings of the International Conference on Service-Oriented Computing Workshops, number 6275 in Lecture Notes in Computer Science, pages 335344. Springer, 2010.

[35] A. Vashist, R. Izmailov, K. Manousakis, R. Chadha, C. Chiang, C. Serban, and S. Ali. Towards network invariant fault diagnosis in MANETs via statistical modeling: The global strength of local weak decisions. In Proceedings of the IEEE Network Operations and Management Symposium, pages 981-987, April 2012.

[36] J. Vomlel. Exploiting functional dependence in Bayesian network inference. In Proceedings of the Eighteenth Conference on Uncertainty in Artificial Intelligence, pages 528-535, 2002.

[37] D. Zhou and T.-H. Lai. An accurate and scalable clock synchronization protocol for IEEE 802.11-based multihop ad hoc networks. IEEE Transactions on Parallel and Distributed Systems, 18(12):1797-1808, Dec. 2007.

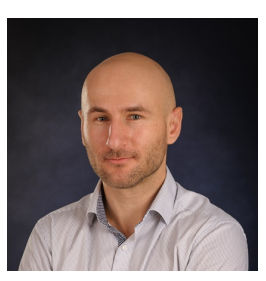

Petr Novotny is a post-doctoral Research Associate in the Department of Computing at Imperial College London. He received the M.Sc. degree in Software Systems Engineering from University College London and the Ph.D. degree in Computing from Imperial College London, and was a visiting scientist at U.S. Army Research Laboratory. His interests are in software engineering and networking.

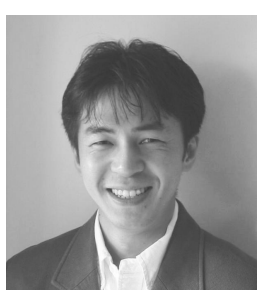

Bong Jun Ko is a research staff member in the Cloud-Based Networking Department at IBM T.J. Watson Research Center. He received the Ph.D. degree in Electrical Engineering from Columbia University in 2006, and the B.S. and M.S. degrees from Seoul National University in South Korea. His research interests include data analytics, cloud computing, mobile networking, and distributed systems.

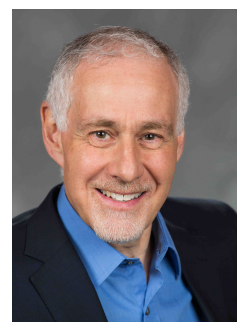

Alexander Wolf is Dean of Engineering and Distiguished Professor of Computer Science and Computer Engineering at the University of California, Santa Cruz, and Professor of Computing at Imperial College London. He received the B.A. degree from the City University of New York, and the M.S. and Ph.D. degrees from the Department of Computer Science at the University of Massachusetts at Amherst. He was previously the C.V. Schelke Chair at the University of Colorado at Boulder, and a member of the technical staff at AT\&T Bell Laboratories. His research interests span the areas of distributed systems, networking, and software engineering. Wolf is a Fellow of the IEEE, ACM, and BCS. 